\title{
Evolution of instabilities in filament buckling processes
}

\author{
A. G. Monastra, ${ }^{1,2,{ }^{*}}$ M. F. Carusela,,${ }^{1,2}$ G. van der Velde, ${ }^{3}$ M. V. D'Angelo, ${ }^{4,2}$ and L. Bruno ${ }^{3,2}$ \\ ${ }^{1}$ Instituto de Ciencias, Universidad Nacional de Gral. Sarmiento, Los Polvorines, Buenos Aires, Argentina \\ ${ }^{2}$ Consejo Nacional de Investigaciones Científicas y Técnicas, Argentina \\ ${ }^{3}$ Departamento de Física \& IFIBA-CONICET, Facultad de Ciencias Exactas y Naturales, Universidad Nacional de Buenos Aires, Argentina \\ ${ }^{4}$ Grupo de Medios Porosos, Facultad de Ingeniería, Universidad Nacional de Buenos Aires, Argentina
}

(Received 9 January 2019; published 25 March 2019)

\begin{abstract}
In this work we study the dynamical buckling process of a thin filament immersed in a highly viscous medium. We perform an experimental study to track the shape evolution of the filament during a constant velocity compression. Numerical simulations reproduce the dynamical features observed from the experimental data and allow quantifying the filament's load. We observe that both the filament's load and the wave number evolve in a stepwise manner. In order to achieve a physical insight of the process, we apply a theoretical model to describe the buckling of a filament in a viscous medium. We solve a hydrodynamic equation in terms of normal modes for clamped-clamped boundary conditions and constant applied load. We find a good agreement between experimental data and simulations, suggesting that the proposed mechanistic model captures the essential features underlying the dynamical buckling process.
\end{abstract}

DOI: 10.1103/PhysRevE.99.033004

\section{INTRODUCTION}

The buckling of filaments under compression in highly viscous media is a phenomenon of interest in numerous applications in physics, biology, medicine, and engineering and involves a wide range of spatial and temporal scales. Examples include the manufacturing of fiber-reinforced composites $[1,2]$, the mechanics of biological polymers [3-5], the motility of microorganisms [6,7], and the movement of cytoskeleton's microtubules [8]. Polymeric solutions allow us to improve the displacement in enhanced oil recovery [9] and fibers are used to prevent proppant flow back in hydraulic fracturing [10] in the oil industry, whereas optical fibers are employed as sensors for measurements in groundwater [11]. In all these processes, the movement of the flexible filaments in a confined environment can produce their entrapment or buckling.

The buckling process is an instability that occurs when a compressing force is applied to the end of a slender rod. If the applied force exceeds a critical value $[12,13]$, then the originally straight filament collapses and buckles. The Euler or critical force depends on the rod geometry and its elasticity. For a clamped-clamped filament its value is

$$
P_{c}=4 \pi^{2} \frac{E I}{L^{2}},
$$

where $E, I$, and $L$ are the filament's Young modulus, second moment of area of cross section, and length, respectively.

Whereas the critical force is independent of the environment where the buckling takes place, the timescale of the subsequent filament deformation strongly depends on the surrounding viscosity [14]. Thereby, the time course of the shape evolution will be slower the more viscous the fluid.
As mentioned, the motion of filaments in viscous media is present in several natural phenomena and industrial applications. Typically, in these cases, the applied load is not constant and thus an equilibrium situation is not attained. For this reason it is of great interest to understand the conditions under which a flexible filament undergoes deformation, as well as its characteristic shape and amplitude. Such deformation could be responsible, for example, for the entrapment of the filament, preventing its transport through the medium.

In this work we register the buckling of a thin rod when it is compressed in a very viscous medium, i.e., glycerol, at constant speed. We recover the filament shapes from the movies and compute the wave number as the deformation evolves for different compressing speeds. To relate these shapes with the load, we develop a numerical simulation of the process and obtain a relationship between the wave number and the load. To achieve a physical understanding, we also develop a mechanical theoretical model of a filament under a constant load. This model is based on normal modes with characteristic wave numbers that are directly related to the compressing force.

\section{MECHANICAL MODEL}

The configuration of a uniform slender semiflexible filament is determined by the position of its neutral axis $\mathbf{r}(l)$, with $l$ a curvilinear coordinate along the filament varying from 0 to $L$ (unstressed length). The configuration has elastic and bending energies given by:

$$
\begin{gathered}
V_{E}=\frac{1}{2} E A \int_{0}^{L} \varepsilon^{2}(l) d l, \\
V_{B}=\frac{1}{2} E I \int_{0}^{L} \mathcal{C}^{2}(l)[1+\varepsilon(l)]^{2} d l,
\end{gathered}
$$


respectively, in terms of the strain $\varepsilon(l)=\left|\mathbf{r}^{\prime}(l)\right|-1$ and curvature $\mathcal{C}(l)=\left|\mathbf{r}^{\prime}(l) \times \mathbf{r}^{\prime \prime}(l)\right|\left|\mathbf{r}^{\prime}(l)\right|^{-3}$ of the filament, where the primes indicate derivative with respect to the coordinate $l$. The other parameters are the Young modulus $E$, the area $A$, and the second moment of area $I$ of the cross section of the filament.

Using a variational principle for both potential energies, the forces $\mathbf{f}_{E}(l)$ and $\mathbf{f}_{B}(l)$ per unit length on an infinitesimal element of the filament can be obtained. The full expressions are rather complicated, especially for the bending term, involving up to the fourth spatial derivative of the position $\mathbf{r}(l)$.

We also take into account a drag force as the filament moves in a homogeneous viscous medium. The precise fluid dynamics is beyond the scope of this work, although we consider a low-Reynolds-number regime. Therefore, the drag force acting on each element is directly proportional to its velocity $\mathbf{f}_{\text {vis }}(l)=-c \dot{\mathbf{r}}(l)$, where the dot accounts for time derivative. The drag coefficient per unit length $c$ is proportional to the dynamical viscosity and also depends on the geometry of the filament.

In an overdamped regime, the inertia term can be neglected and, finally, the equation of motion for an infinitesimal segment is given by

$$
\mathbf{f}_{E}(l)+\mathbf{f}_{B}(l)+\mathbf{f}_{\mathrm{vis}}(l)+\mathbf{f}_{\mathrm{ext}}(l)=0,
$$

where $\mathbf{f}_{\text {ext }}$ denotes applied external forces. The solution is determined by the initial configuration of the filament and its boundary conditions. For small deviations from the straight shape and constant compressing load $P$, Eq. (4) has a general analytic solution

$$
\delta y(l, t)=\sum_{n=1}^{\infty} \sum_{\sigma} a_{n}^{\sigma} \Psi_{n}^{\sigma}(P, l) \exp \left(\Gamma_{n}^{\sigma} t\right)
$$

in terms of symmetric $(\sigma=+1)$ and antisymmetric $(\sigma=$ -1) normal modes $\Psi_{n}^{\sigma}$, which depend parametrically on $P$ [15]. For a force larger than $P_{c}$, there is at least one mode which exponentially grows in time $\left(\Gamma_{n}^{\sigma}>0\right)$. In this case the corresponding mode $\Psi_{n}^{\sigma}$ is a linear superposition of two sinusoidal waves with definite wave numbers,

$$
\kappa_{ \pm}=\sqrt{\frac{p \pm \sqrt{p^{2}-4 \gamma}}{2}},
$$

where $p=L^{2} P /(E I)$ and $\gamma=\Gamma c L^{4} /(E I)$. For the clampedclamped boundary condition the values of $\gamma$ as a function of $p$, for each mode $n$ and parity $\sigma$, can be obtained numerically finding the roots of a nonlinear equation [15]. For $P \gg P_{c}$ these values can be approximated by

$$
\gamma_{n}^{\sigma} \approx \frac{p^{2}}{4}-\left(n \pi \sqrt{2 p-4 \pi^{2} n^{2}}+\sqrt{2 p} \epsilon_{n}^{\sigma}\right)^{2},
$$

where $\epsilon_{n}^{\sigma}$ is an oscillating function of $p$, much smaller than 1. Replacing this approximation in Eq. (6), the corresponding wave numbers are

$$
\kappa_{ \pm} \approx \sqrt{\frac{p}{2} \pm \sqrt{2 \pi^{2} n^{2}\left(p-2 \pi^{2} n^{2}\right)} \pm \sqrt{2 p} \epsilon_{n}^{\sigma}} .
$$

Numerically, it turns out that this approximation is very accurate even for values of $P$ near the critical value. As an

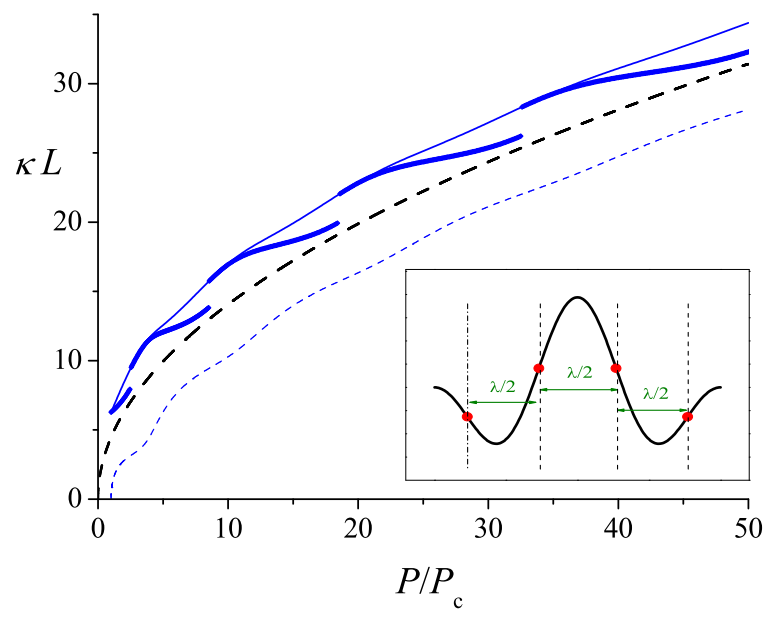

FIG. 1. Wave numbers $\kappa_{-}$and $\kappa_{+}$(dash and continued thin lines) as a function of compressing force for $n=1$ symmetric $(\sigma=+1)$ mode. Thick line: Effective wave number. Thick dash line corresponds to mean wave number $\kappa=\sqrt{p / 2}=\sqrt{2 \pi^{2} P / P_{c}}$. Inset: Sketch of a normal mode and inflection points in circular dots.

example, we plot in Fig. 1 the two wave numbers for $n=1$ symmetric $(\sigma=+1)$ mode, which have the largest growing rate $\Gamma_{n}^{\sigma}$.

For a given mode, if the precise value of the compressing force is unknown, then it would be difficult to determine the two characteristic wave numbers from the inspection of the filament's shape. This is even more difficult if there is a superposition of modes or an arbitrary shape, as it typically occurs in the experiments.

To overcome this limitation we estimate an effective wave number using the following criterion: For a perfect sinusoidal wave, the distance between two consecutive inflection points is trivially a half wavelength (see inset Fig. 1). Therefore, for a particular configuration of the filament, we compute the position of $N$ inflection points, obtaining $(N-1)$ values for a half wavelength. Averaging these values, we compute an average wavelength $\bar{\lambda}$. Finally, we estimate $\kappa_{\text {eff }}=2 \pi / \bar{\lambda}$. This criterion also gives a normal standard error, calculated from the dispersion of the computed wavelengths. In Fig. 1 we plot this effective wave number applied to the theoretical solutions (thick line). We observe discontinuities, given by the appearance of new inflection points with the increasing force.

This approximation may not work properly in the experimental case: Observed amplitudes can be comparable to $L$, boundary conditions are more complex, and compressing force can vary in time and be nonhomogeneous along the filament. However, these expressions give a relation between the compressing load (difficult to measure) and the wave numbers in a filament's shape.

\section{EXPERIMENTS}

The experiments were carried out inside a horizontal glass cell, open at the top, of length $L_{c}=(35.0 \pm 0.1) \mathrm{cm}$, width $W_{c}=(15.0 \pm 0.1) \mathrm{cm}$, and height $H_{c}=(20.0 \pm 0.1) \mathrm{cm}$. The filaments used were acetate strips of rectangular section of width $a=(4.0 \pm 0.1) \mathrm{mm}$, thickness $e=(100 \pm 1) \mu \mathrm{m}$, and length $L=(28.6 \pm 0.1) \mathrm{cm}$. Flat filaments were used 

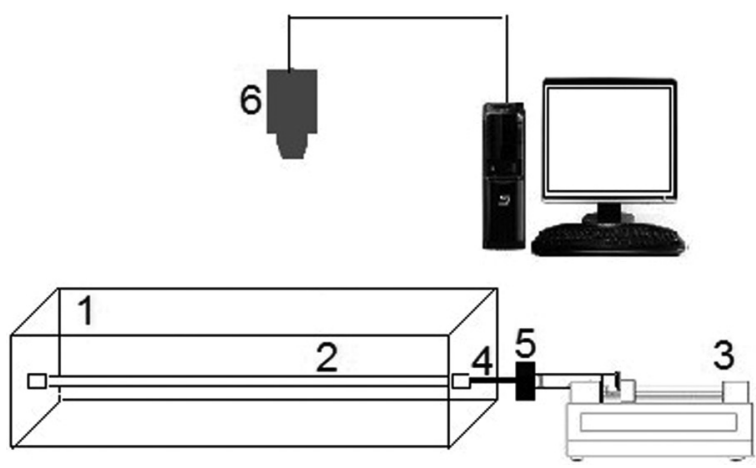

FIG. 2. Schematic view of the experimental set-up: 1, horizontal cell; 2 , filament; 3 , syringe pump; 4, acrylic rod; 5 , piston; 6 , camera.

to reduce possible torsions and to induce deformation in the plane perpendicular to the optical axis of the camera.

The Young's modulus of the filaments was $(2.5 \pm$ 0.5) GPa. This value was estimated using the cantilever method by measuring the deflection of the filament at one of its ends when a transverse force is exerted on the free end.

Each end of the filament was held by two small pieces of acrylic. One of these pieces was fixed to one end of the cell, while the other end was joined, at the opposite side, to an acrylic rod that could be moved inside the cell. The device was uniformly illuminated from the bottom of the cell using a light panel.

The cell was filled with glycerol until the filament was fully covered. The fluid density and viscosity at room temperature were approximately equal to $1.26 \mathrm{~g} / \mathrm{cm}^{3}$ and $1.49 \mathrm{~Pa} \mathrm{~s}$, respectively.

The complete device is shown in Fig. 2.

For compression experiments the filament was placed in the holders, making sure that it was completely stretched and without torsion. Then the filament was compressed either manually or by means of the piston of the syringe pump (Harvard MA 55-52226, Model 22), pushing the movable end of the filament at a constant speed. The advance of the piston was stopped after moving a few centimeters from the initial position to guarantee small amplitude deformations. After the piston arrest, the filament relaxes.

Images were recorded during compression and relaxation using a digital camera, with spatial resolution of $0.17 \mathrm{~mm} / \mathrm{pixel}$ and a frame size of $900 \times 2010$ pixels. Images were acquired at constant time intervals $(1 / 60 \mathrm{~s}$ or $1 / 70 \mathrm{~s})$.

All images were processed after each experiment in order to determine the instantaneous position of the piston and the filament's shape. To this purpose, we adapted the tracking routines described in Ref. [16]. Briefly, points belonging to the filament were manually selected from the initial frame and used by the code as an initial guess for the tracking. Then the intensity profiles in the transverse direction at successive pixels along the filament extent ( $x$ coordinate) were interpolated and the positions of the maximum were computed ( $y$ coordinate). This method provides a subpixel determination of the filament coordinates. The error of the tracking was $0.13 \mathrm{~mm}$. The procedure was repeated automatically for all the following frames in the movie. The compressing velocity was measured by image processing, too, and included into the code in order to determine the edge of the filament at each frame.

\section{NUMERICAL SIMULATION}

There are no analytic solutions for the hydrodynamic equation (4) in the complex experimental situation, where the speed of compression can be controlled but not the compressing force. Nevertheless, the physics of the deformation can be explored by means of a finite-element simulation of the process. To this end, the filament is considered as $N$ equal segments of length $\Delta l=L / N$. The configuration is determined by the $(N+1)$ coordinates $\mathbf{r}_{n}$ of the end points of each segment, where $0 \leqslant n \leqslant N$. In terms of these coordinates, the elastic and bending potential energies can be written as:

$$
\begin{aligned}
V_{E} & =\frac{1}{2} \frac{E A}{\Delta l} \sum_{n=0}^{N-1}\left(\left|\mathbf{r}_{n+1}-\mathbf{r}_{n}\right|-\Delta l\right)^{2} \\
V_{B} & =\frac{E I}{\Delta l} \sum_{n=1}^{N-1}\left[1-\frac{\left(\mathbf{r}_{n+1}-\mathbf{r}_{n}\right) \cdot\left(\mathbf{r}_{n}-\mathbf{r}_{n-1}\right)}{\left|\mathbf{r}_{n+1}-\mathbf{r}_{n}\right|\left|\mathbf{r}_{n}-\mathbf{r}_{n-1}\right|}\right] .
\end{aligned}
$$

Other expressions can be considered, depending on how the discrete derivatives are defined, although these expressions converge to Eqs. (2) and (3) in the limit $\Delta l \rightarrow 0$. Deriving the potential energies with respect to the coordinate $\mathbf{r}_{n}$, elastic and bending forces are obtained,

$$
\begin{aligned}
& \mathbf{F}_{n}^{E}=-\frac{\partial V_{E}}{\partial \mathbf{r}_{n}}, \\
& \mathbf{F}_{n}^{B}=-\frac{\partial V_{B}}{\partial \mathbf{r}_{n}},
\end{aligned}
$$

which are applied to the corresponding bead $n$. The viscous force is given by $\mathbf{F}_{n}^{\mathrm{vis}}=-c \Delta l \dot{\mathbf{r}}_{n}$ for $1 \leqslant n \leqslant(N-1)$. For the end beads $n=0$ and $n=N$, the viscous force is $\mathbf{F}_{n}^{\mathrm{vis}}=-c(\Delta l / 2) \dot{\mathbf{r}}_{n}$, corresponding to the drag on both ending semisegment.

Neglecting again the inertia, we arrive at $(N+1)$-coupled first-order differential equations,

$$
\dot{\mathbf{r}}_{n}=\frac{\alpha_{n}}{c \Delta l}\left(\mathbf{F}_{n}^{E}+\mathbf{F}_{n}^{B}+\mathbf{F}_{n}^{\mathrm{ext}}\right)
$$

where $\alpha_{n}=1$ for $1 \leqslant n \leqslant(N-1)$ and $\alpha_{n}=2$ for $n=0$ and $n=N$. Given the parameters $E, A, I, c$, and boundary conditions, these equations are integrated numerically from a given initial configuration. For the values of the experimental velocities, $N=150$ segments was enough to capture the shape and observed wave numbers.

The chosen initial configuration is a cosine shape which best approaches the observed relaxed experimental shape. Both ends fulfill a clamped condition. We implement it numerically in the following way: We force the first bead to move horizontally at constant velocity while the last one is fixed. For the clamped-clamped boundary condition we constrain the second and penultimate bead to move only horizontally. 


\section{RESULTS AND DISCUSSION}

\section{A. Estimation of the drag coefficient}

Our model depends on several parameters, most of which can be estimated quite accurately from tabulated values or direct measurements. However, this is not the case for the drag coefficient $c$, which has complex dependencies with the shape and inclination of the body, the Reynolds number of the flow, the roughness of the surface, and the viscosity of the fluid, to mention a few.

In the experiments we observe two clearly different stages of the buckling process. The first one is related to the dynamics due to the pushing constant velocity of the piston (compression). The second one corresponds to the dynamics taken place when the piston stops and the filament shape relaxes (relaxation). The dynamical response of the filament shows to be very sensitive to the drag coefficient, which plays a key role in both compression and relaxation stages. Consequently, it is mandatory to get a precise value of this parameter to achieve a more accurate description of the experimental data.

From the relaxation stage we can extract information about the characteristic time of this process, which is directly related to the drag coefficient $c$. For this purpose, we carry out the following procedure. We track the shape of the filament in the relaxation regime for different frames of one movie at arbitrary times. Then we simulate the corresponding shapes at the same times for a given $c$ and we take the euclidean distance between the simulated and experimental contours. We repeat this procedure adjusting $c$ until the distance becomes minimum (within an uncertainty interval), obtaining an optimum $c=(91 \pm 1)$ dyn $\mathrm{s} / \mathrm{cm}^{2}$. We check that this value is independent of the selected times or the chosen experimental set. Then this value is used for the simulations in the compression stage.
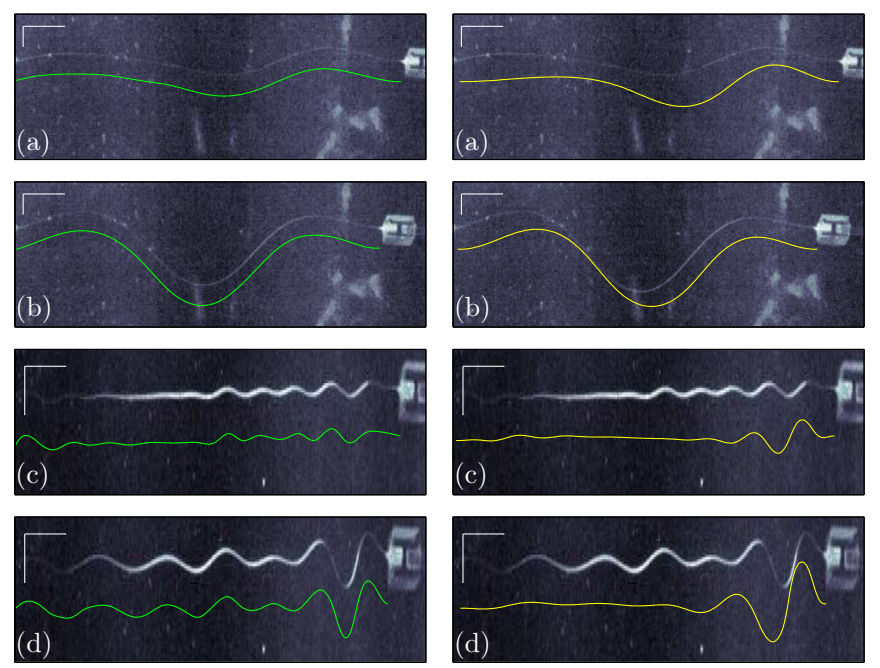

FIG. 3. Snapshots of two experiments. [(a) and (b)] $v_{0}=0.062$ $\mathrm{cm} / \mathrm{s}, t=10.0 \mathrm{~s}$, and $33.3 \mathrm{~s}$, respectively. [(c) and (d) $] v_{0}=5 \mathrm{~cm} / \mathrm{s}$, $t=0.0625 \mathrm{~s}$, and $0.25 \mathrm{~s}$. The experimental tracking [left panels (green)] and the numerical simulation [right panels (yellow)] are plotted as thin lines $1 \mathrm{~cm}$ below the filament to facilitate visual inspection. Horizontal and vertical scale bars represent $3 \mathrm{~cm}$ and 1 $\mathrm{cm}$, respectively.
TABLE I. Experimental and simulated effective wave number $\kappa$ for snapshots shown in Fig. 3

\begin{tabular}{lcc}
\hline \hline Data & $\kappa_{\exp } L$ & $\kappa_{\text {sim }} L$ \\
\hline (a) & $13.8 \pm 0.5$ & $12.6 \pm 3.5$ \\
(b) & $10.8 \pm 1.6$ & $11.9 \pm 1.3$ \\
(c) & $62 \pm 18$ & $53.4 \pm 7.9$ \\
(d) & $46 \pm 15$ & $34.6 \pm 5.0$ \\
\hline \hline
\end{tabular}

\section{B. Constant speed compression}

We analyze in detail two experiments performed at two different velocities. Initially, the filament is straight up to the tracking error. The boundary conditions are clamped-clamped (Fig. 3).

We perform numerical simulations of the buckling process for the two experimental velocities. We choose an initial configuration with an amplitude of $1 \mathrm{~mm}$, which is much smaller than the length of the filament. In Fig. 3 we show experimental snapshots at two different times for each velocity, where we also plot the shapes obtained with the tracking procedure (green) and with the simulations (yellow). Some differences can be noticed, mostly in the positions of the maxima and minima, although the amplitudes and shapes are rather similar. We do not assign these differences to a failure of the physical model but to a sensitivity to initial configuration of the filament, as assessed numerically.

Applying the inflection point criterion described above to the experimental and simulated shapes, we compute the effective wave number $\kappa$ (see Table I). A good agreement between simulations and experiments is found for high and low velocities.

For the simulations, we also compute the longitudinal forces at the fixed and moving ends. A representative result is plotted in Fig. 4.

The observed behavior is qualitatively similar for all speeds and initial shapes, provided that the initial amplitude is much smaller than $L$. For short times (notice the logarithmic scale in time), the forces display a very rapid growth provided by an isostatic compression. During this period $(t<0.03 \mathrm{~s}$ in

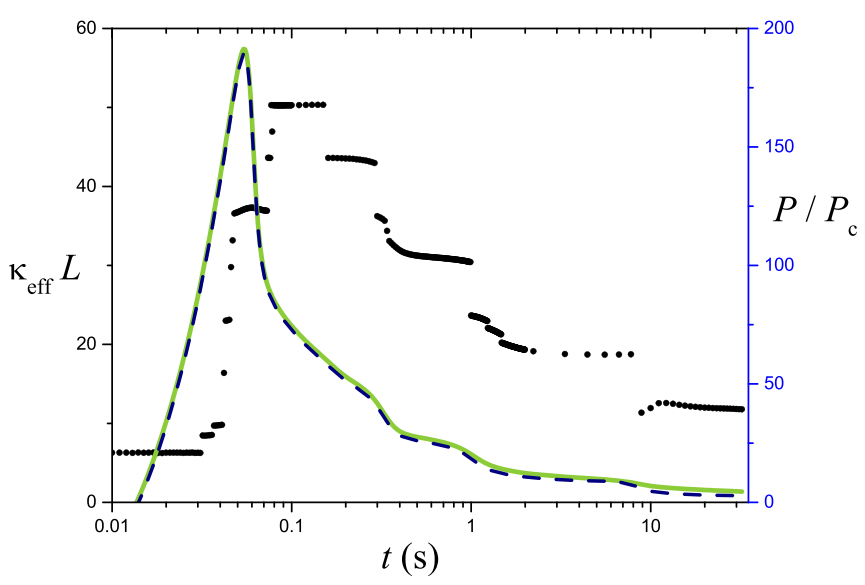

FIG. 4. Forces computed at the moving (continuous line) and fixed (dash line) ends of the filament, and effective wave number (dots) as a function of time, for $v_{0}=0.062 \mathrm{~cm} / \mathrm{s}$. 


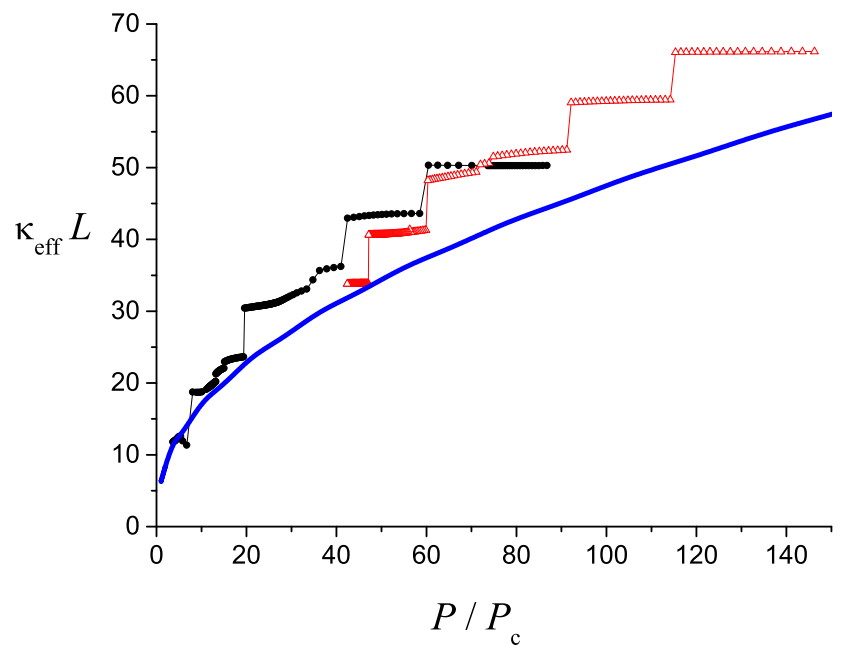

FIG. 5. Wave number as a function of average force for the two simulations of the experiments at $v_{0}=0.062 \mathrm{~cm} / \mathrm{s}$ (circles) and $v_{0}=5.0 \mathrm{~cm} / \mathrm{s}$ (triangles). The continous curve corresponds to the theoretical value $\kappa_{+}$for the symmetric mode $n=1$.

the case shown), the filament's shape and amplitude do not vary, although the force increases several orders of magnitude. This increasing force excites high-wave-number modes that grow exponentially in time. At some point the force achieves a maximum and it decays rapidly while the amplitude increases. Later, as the force continues to decrease, the wave numbers go down in a stepwise manner. This nonmonotonic behavior of the wave numbers is a time-shift delay with respect to the compressing force, suggesting a memory effect due to the response timescales of the different modes. In other words, there is a kind of hysteresis in the dynamical behavior of the system.

In Fig. 5 we plot the wave number as a function of the force for both theory and simulations. For the theoretical case, we plot $\kappa_{+}$for the first symmetric mode $n=1$. For simulations we only plot data after the maximum force was attained to avoid the isostatic compression stage. Even for velocities with two orders of magnitude of difference, there is an overlap in the intermediate range of forces, indicating a similar underlying mechanism. The fact that simulations are slightly above theoretical curves is a sign of the hysteresis mentioned above.

Although our theoretical model is based on a constant compressing load, which is not the case of the experiments, it gives us a physical understanding of the relation between the wave numbers obtained experimentally and the load, which is a great challenge to measure in experimental conditions.

\section{CONCLUSIONS}

In this paper we studied the dynamics of buckling instabilities taking place when a thin filament immersed in a very viscous fluid is compressed. Particularly, we explored the buckling evolution during a constant velocity compression. We determined an effective wave number to characterize the shape of the filament and its evolution during the compression. By means of numerical simulation, we were able to relate the applied compressing force with the wave number for both high and low velocities. This is compatible with the theoretical predictions for constant loads, pointing out a similar underlying mechanism. Increasing the compressing speed results in higher forces and larger wave numbers. After the maximum force is reached, forces and wave numbers evolve in a stepwise manner.

Our numerical model enables the study of the time evolution and relation between the compressing force and the wave number. The simulations also suggest a memory effect in the dynamics, although this does not affect the general conclusions obtained in this work.

These results are interesting since they allow us to estimate the values of the forces acting on a filament in a noninvasive way, just measuring the wavelength from a filament's image. This could be very useful for instance in the case of microtubules within cells, whose buckling indicates internal forces controlling important mechanical processes in the intracellular medium.

\section{ACKNOWLEDGMENTS}

M.F.C. and A.G.M. acknowledge Grant No. 14420140100013CO PIO-CONICET (AR).
[1] K. Yasuda, N. Mori, and K. Nakamura, Int. J. Eng. Sci. 40, 1037 (2002).

[2] D. Y. Yoo, S. Kim, G. J. Park, J. J. Park, and S. W. Kim, Composite Structures 174, 375 (2017).

[3] M. C. Lagomarsino, I. Pagonabarraga, and C. P. Lowe, Phys. Rev. Lett. 94, 148104 (2005).

[4] A. Ghosh, J. Samuel, and S. Sinha, Phys. Rev. E 76, 061801 (2007).

[5] M. Emanuel, H. Mohrbach, M. Sayar, H. Schiessel and I. M. Kulić, Phys. Rev. E 76, 061907 (2007).

[6] C. Lowe, Philos. Trans. R. Soc. Lond. B 358, 1543 (2003).

[7] B. Chaban, H. Velocity Hughes, and M. Beeby, Semin. Cell Dev. Biol. 46, 91 (2015).

[8] C. P. Brangwynne, F. C. MacKintosh, S. Kumar, N. A. Geisse, J. Talbot, L. Mahadevan, K. K. Parker, D. E. Ingber, and D. A. Weitz, J. Cell Biol. 173, 733 (2006).
[9] C. Xie, W. Lv, and M. Wang, J. Pet. Sci. Eng. 161, 683 (2018).

[10] F. Liang, M. Sayed, G. A. Al-Muntasheri, F. F. Chang, and L. Li, Petroleum 2, 26 (2016).

[11] J. Selker, N. van de Giesen, M. Westhoff, W. Luxemburg, and M. B. Parlange, Geophys. Res. Lett. 33, L24401 (2006).

[12] L. D. Landau and E. M. Lifshitz, Theory of Elasticity, Vol. 7, Course of Theoretical Physics (Elsevier, New York, 1986).

[13] S. P. Timoshenko and J. M. Gere, Theory of Elastic Stability (McGraw-Hill, New York, 1963).

[14] J. Howard, Mechanics of Motor Proteins and the Cytoskeleton (Sinauer, Sunderland, MA, 2001).

[15] A. G. Monastra, M. F. Carusela, M. V. D’Angelo, and L. Bruno, J. Phys.: Conf. Ser. 1012, 012010 (2018).

[16] C. Pallavicini, V. Levi, D. E. Wetzler, J. F. Angiolini, L. Benseñor, M. A. Desposito, and L. Bruno, Biophys. J. 106, 2625 (2014). 